\section{Kramer}

\section{Plasmapheresis in the ICU setting - Indications and side effects}

Summary The last two decades have seen a marked change in the role of plasmapheresis in the intensive care unit (ICU). Initially regarded as highly effective in treating virtually any immunological disease, insights in pathophysiology and results from controlled trials have left

Eingegangen: 2. März 1998

Akzeptiert: 8. April 1998

Dr. L. Kramer (凶)

Intensivstation

Universitätsklinik für Innere Medizin IV

Währinger Gürtel 18-20

A-1090 Wien

Österreich

E-mail: ludwig.kramer@akh-wien.ac.at

\title{
Indikationen und Komplikationen der Plasmapherese im Rahmen der Intensivmedizin
}

only a few indications validated. To date, plasmapheresis is indicated for acute treatment of severe immunological disorders either unresponsive to immunosuppression or requiring large volumes of human plasma for therapy. In the future, more specific and less cumbersome methods of immunosuppression and immunomodulation might further limit indications of plasmapheresis. In this review, current indications and side-effects of plasmapheresis in the ICU setting are summarized.

Key words Extracorporeal renal support - plasmapheresis indications - complications

Zusammenfassung Die Rolle der Plasmapherese in der Intensivmedizin hat sich gewandelt. Nach einer initial unkritischen Verwendung als „moderner Aderlaß“ zur Behandlung vieler akuter immunologischer Erkrankungen wurde das Indikations- spektrum im Verlauf der letzten zwei Dekaden reduziert. Heute gelten nur wenige schwere akute, meist immunologische Erkrankungen als gesicherte Indikation zur Plasmapherese, welche überwiegend als Überbrückung bis zum Wirkungsbeginn immunsuppressiver Medikamente oder zur zusätzlichen Plasmazufuhr eingesetzt wird. Für die Zukunft ist zu erwarten, daß die Plasmapherese durch spezifische und nebenwirkungsarme extrakorporale Methoden sowie durch verbesserte Methoden der Immunsuppression und -modulation weiter zurückgedrängt wird. Der vorliegende Artikel gibt einen Überblick über heutige Indikationen und Komplikationen der Plasmapherese im Rahmen der Intensivmedizin.

\section{Schlüsselwörter Extrakorporale} Nierenersatzverfahren Plasmapherese - Indikationen Komplikationen

\section{Einleitung}

Die Plasmapherese [„,Plasmaentfernung“] wurde bereits zu Beginn dieses Jahrhunderts u. a. durch Abel beschrieben und zur Gewinnung von Tetanus-Antikörpern verwendet [1]. Erst seit den 70er Jahren wird die kontinuierliche maschinelle Plasmapherese auch allgemein therapeutisch eingesetzt [2]. In einer ersten unkritischen Phase wurde eine Vielzahl an zumeist seltenen und therapierefraktären Erkrankungen als Indikationen zur Plasmapherese genannt; die Mehrzahl der wenigen kontrollierten Studien verlief al- lerdings negativ [3]. Derzeit gelten nur wenige akute, meist immunologische Erkrankungen als gesicherte Indikationen zur Plasmapherese, welche überwiegend als Überbrückung bis zum Wirkungsbeginn immunsuppressiver Medikamente eingesetzt wird. Meist ist ein frühzeitiger Einsatz entscheidend zur Verhinderung irreversibler Organschäden. Obgleich nicht alle Indikationen der Plasmapherese kritische, ,intensivpflichtige“"Erkrankungen sind, sollten Intensivmediziner aufgrund der häufigen Durchführung des Verfahrens auf Intensiv- und Akutdialysestationen über Indikationen und potentielle Komplikationen informiert sein. 
Berücksichtigt man, daß die Plasmapherese relativ häufig zu Komplikationen führt [4] und daß etliche immunologische Indikationen mit moderneren Verfahren wie der Immunadsorption (Bindung von IgG an Adsorptionssäulen), Immunsuppressiva, Infusion von Immunglobulinen oder in Zukunft möglicherweise durch spezifische Immuntherapie effizienter und nebenwirkungsärmer behandelt werden können [5], stellt die Vermeidung überflüssiger oder durch spezifische Methoden zu ersetzender Behandlungen die wichtigste Prophylaxe von Komplikationen dar.

\section{Pathophysiologische Grundlagen}

\section{Substanzentfernung}

Häufigstes Ziel der Plasmapherese ist die Entfernung hochmolekularer Substrate wie Immunglobuline (IgG, IgM), Immunkomplexe und proteingebundener Toxine aus dem Plasma [6] (Tabelle 1). Die Porengröße der verwendeten Membranen liegt zwischen 0,2 und 0,6 $\mu \mathrm{m}$, entsprechend einer molekularen Ausschlußgröße von ca. $3 \times 10^{6} \mathrm{D}$ [6]. Im Plasma gelöste Proteine werden unselektiv entfernt, was ein Absinken des kolloidosmotischen Drucks bewirkt und eine Kolloidsubstitution notwendig macht. Genaugenommen sollte zwischen Plasmapherese (Austausch von Plasma gegen Albumin oder andere Kolloide) und therapeutischem Plasmaaustausch (Austausch gegen Plasma) stets unterschieden werden.

Je höher der im Plasma zirkulierende Anteil einer Substanz ist, um so effizienter ist die einzelne Plasmapherese hinsichtlich ihrer Entfernung. Da aber z. B. IgG durch eine einmalige Plasmapherese des gesamten Plasmavolumens lediglich auf ca. 37\% des Ausgangswertes absinken [4] und durch Rückverteilung und Neusynthese bereits nach $24 \mathrm{~h}$ auf fast $70 \%$ des Ausgangswert ansteigen, sind in der Regel mehrere engmaschige und möglichst großvolumige Plasmaaustauschbehandlungen in Kombination mit Immunsuppression nötig. Die Effizienz der Immunapherese unter Verwendung von Anti-human-IgG-Säulen ist mit 80-90\% IgG-Entfernung wesentlich höher [7].

Tab. 1 Molekülgröße, intravaskulärer Anteil und Siebkoeffizient* von Albumin und Immunglobulinen [6]

\begin{tabular}{lllll}
\hline Substanz & $\begin{array}{l}\text { Molekular- } \\
\text { gewicht } \\
(\mathrm{kD})\end{array}$ & $\begin{array}{l}\text { Intra- } \\
\text { vaskulär } \\
(\%)\end{array}$ & $\begin{array}{l}\text { HWZ } \\
\text { (Tage) }\end{array}$ & $\begin{array}{l}\text { Sieb- } \\
\text { koeffizient }\end{array}$ \\
\hline Albumin & 69 & 40 & 19 & $>0,95$ \\
IgG & 180 & 50 & 21 & $>0,90$ \\
IgA & 150 & 50 & 6 & 0,85 \\
IgM & 900 & 80 & 5 & 0,80 \\
\hline
\end{tabular}

* Siebkoeffizient bei $100 \mathrm{ml} / \mathrm{min}$ Blutflußrate und $40 \mathrm{mmHg}$ Transmembrandruck
Immunmodulatorischer Effekt

Neben der Entfernung von humoralen Substanzen wird auch eine unspezifische Verbesserung der Funktion des reticuloendothelialen Systems (RES) angestrebt [8]. Dieser wird mit einer „Entlastung“ des RES durch die extrakorporale Entfernung von Immunglobulinen und Immunkomplexen erklärt.

\section{Substanzzufuhr}

Bei einigen Indikationen beruht der empirische Behandlungserfolg nicht nur auf Entfernung, sondern auch auf der Zufuhr von Plasmafaktoren. Hier handelt es sich v. a. um die thrombotisch-thrombozytopenische Purpura (Moschkowitz) und um die fulminante Meningokokkensepsis $[9,10]$.

\section{Perioperative Plasmapherese}

In dieser neuen Indikation wird vor herzchirurgischen Eingriffen thrombozytenreiches Plasma durch eine Plasmapherese entnommen und nach Abgehen vom kardiopulmonalen Bypass reinfundiert [11].

\section{Spezifische Indikationen der Plasmapherese:}

Renale Indikationen

Renale Erkrankungen, häufig in Form renaler Beteiligung bei Systemerkrankungen, stellen die häufigste Indikation zum Einsatz der Plasmapherese dar. Für diese Indikationsgruppe sind auch Anzahl und Qualität kontrollierter Studien am höchsten.

\section{Rapid-progressive Glomerulonephritis (RPGN)}

Unter diesem klinischen Syndrom werden ätiologisch unterschiedliche akute Glomerulonephritiden zusammengefaßt. Die gemeinsame klinische Endstrecke sind eine akute Verschlechterung der Nierenfunktion meist über wenige Tage bis Wochen und histologische Zeichen einer nekrotisierenden Glomerulonephritis, mit Halbmondbildung und meist extrakapillärer Proliferation [3, 12]. Die Intensität der Plasmapherese scheint mit der klinischen Wirkung zu korrelieren, weshalb tägliche Behandlungen mit Austausch des gesamten Plasmavolumens (beim Erwachsenen ca. 5\% des Körpergewichts) empfohlen werden [3].

- Pauci-immune RPGN: Dies ist die häufigste Form renaler Beteiligung bei Wegener'scher Granulomatose, Panarteritis nodosa, systemischer Vasculitis oder mi- 
kroskopischer Polyangiitis. Meist sind zirkulierende anticytoplasmatische Antikörper (ANCA) nachweisbar. Aufgrund der hohen Ansprechrate auf Immunsuppression ist die Plasmapherese erst bei manifestem Nierenversagen indiziert und durch Ergebnisse kontrollierter Studien gesichert (Remissionsrate bereits dialysepflichtiger Patienten $76 \%$ vs. $58 \%$; p=0,02) [12]. Tägliche oder zumindest 4 Behandlungen pro Woche sollten angestrebt werden.

- Antibasalmembran-Antikörper-RPGN: Antibasalmembran-Antikörper sind pathogen und auch im Tiermodell nephrotoxisch. Durch Einführung von Plasmapherese und Immunsuppression in den späten 70er Jahren konnte die Überlebensrate dieser bislang meist fatalen Erkrankung dramatisch verbessert werden [12]. Acht bisherige Arbeiten, darunter eine kontrollierte Studie ergaben, daß tägliche Plasmapheresen für 14 Tage in Kombination mit konventioneller Immunsuppression die Inzidenz terminaler Niereninsuffizienz, vor allem bei frühzeitigem Beginn (Kreatinin $<6.8 \mathrm{mg} / \mathrm{dl}$ ), deutlich reduzieren kann [12]. Bei bereits länger manifestem oligurischem Nierenversagen ist die Plasmapherese meist wirkungslos hinsichtlich renaler Verbesserung und sollte auf pulmonale Blutungen beschränkt werden.

- Fokal-nekrotisierende GN ohne Basalmembran-AK: Plasmapherese erbrachte zusätzlich zur immunsuppressiven Therapie (Prednisolon, Cyclophosphamid, Azathioprin) einen mäßigen aber signifikanten renalen $\mathrm{Be}-$ nefit gegenüber alleiniger Immunsuppression [13].

\section{Lupus-Nephritis}

Obwohl unkontrollierte Studien und Einzelfälle eine günstige Wirkung der Entfernung von Immunkomplexen nahelegen, bieten kontrollierte Studien keinen Anhaltspunkt für eine Rolle der Plasmapherese bei Lupus-Nephritis [14]. Die noch nicht im Detail publizierte multizentrische Lupus Plasmapheresis Study Group (LPSG) - Studie erbrachte keinen Unterschied in Mortalität und Nierenfunktion zwischen Immunsuppression alleine und zusätzlicher Plasmapherese bei SLE $[15,16]$. Die Indikationen zur Plasmapherese dürften sich daher auf schwere fulminante Nephritis, cerebrale Vasculitis, pulmonale Blutungen und TTP (s.u.) beschränken $[12,17]$.

\section{Myelom-Leichtkettennephropathie}

Zwar können Dauer und Schweregrad eines akuten Nierenversagens durch Entfernung von Leichtkettenproteinen mittels Plasmapherese reduziert werden, dies erscheint allerdings gegenüber dem Ansprechverhalten auf Chemotherapie von nur unwesentlicher prognostischer Bedeutung [18]. Über prophylaktische Plasmapherese liegen nicht genügend Daten vor [19].

\section{Kryoglobulinämie}

Kryoglobuline sind kältepräzipitierende Immunglobuline. Die in drei Typen (I-III) eingeteilte Erkrankung führt häufig zu einer milden membranoproliferativen GN mit subendothelialen Ablagerungen von Kryoglobulinen. Da lediglich unkontrollierte Studien über Plasmapherese bei gemischter (Typ II) Kryoglobulinämie vorliegen, kann ihre Wirkung nicht sicher beurteilt werden [20]. Allerdings dürfte die bei ca. $2 / 3$ der Patienten berichtete Verbesserung vorwiegend bei klinisch aktiver Form und frischen renalen Läsionen (Biopsie) in Kombination mit Immunsuppression zu erwarten sein [3]. Nach heutigem Wissen erscheint primär eine kausale Therapie der chronischen Hepatitis C zielführend [21].

\section{Hämatologische Indikationen}

- Thrombotisch-thrombozytopenische Purpura (TTP): Diese auch als thrombotische Mikroangiopathie bezeichnete Erkrankung ist ein akutes Krankheitsbild mit hoher Letalität, bei der es durch Thrombosierung in der Mikrozirkulation zu Thrombopenie, schwerer Hämolyse und Multiorganversagen kommt. Das Spektrum klinischer Symptome reicht von Hämolyse mit akutem Nierenversagen (hämolytisch-urämisches Syndrom, HUS) bis zur TTP mit hohem Fieber, Purpura, Thrombopenie, neurologischer Symptomatik und Multiorganversagen mit bis zu 90\% Letalität. Aus Platzgründen wird hier nicht näher auf die Pathogenese eingegangen. Die Überlegenheit der Plasmapherese gegenüber alleiniger Plasmainfusion ist gesichert; derzeit können bis über $90 \%$ der Patienten Remission erreichen [22]. Die Plasmapherese sollte durch 5 Tage gegen FFP erfolgen; in therapierefraktären Fällen ist die Splenektomie zu erwägen.

- Hyperviskositätssyndrom bei M. Waldenström: Neurologische Symptome (Schwindel, Kopfschmerzen, Störung der Bewußtseinslage) bei mehr als doppelt erhöhter Plasmaviskosität stellen eine Akutindikation zur Plasmapherese dar [23]. IgM ist vorwiegend intravaskulär vorhanden und kann durch 2-3 Plasmapheresen effizient abgesenkt werden.

- Therapiefraktäre immunhämolytische Anämie und Vorbereitung von Akutoperationen bei Hemmkörper-Hämophilie sind seltene Indikationen zur Plasmapherese [6].

\section{Neuromuskuläre Erkrankungen}

- Myasthenia gravis: Die Entfernung von Acetylcholinrezeptoren-Antikörper kann bei myasthenischer Krise eine respiratorische Insuffizienz beheben und die motorische Situation verbessern [24]. Eine rezente Vergleichsstudie von Plasmapherese und Immunglobulinen 
$(0.4 \mathrm{~g} / \mathrm{kg}$ täglich für 3 oder 5 Tage $)$ zeigte an 87 Patienten mit myasthenischer Krise keine wesentlichen Unterschiede im Therapieeffekt bei höherer Nebenwirkungsrate der Plasmapherese [25]. Das Lambert-EatonSyndrom hat eine geringere Ansprechrate [26].

- Polyradikulitis Guillain-Barré (Guillain-Barré-Syndrom): Die Behandlung des Guillain-Barré-Syndroms mit Plasmapherese verkürzt offenbar durch Entfernung von Anti-Myelin-Antikörpern die Dauer der neurologischen Beeinträchtigung signifikant [27, 28]. Allerdings konnte dieser Effekt auch durch hochdosierte Immunglobuline (IG) erreicht werden [29]. Eine jüngst publizierte multizentrische Studie verglich Plasmapherese, Immunglobuline und die Kombination aus Plasmapherese mit anschließender Immunglobulininfusion an 383 Patienten mit Guillain-Barré-Syndrom innerhalb der ersten beiden Wochen nach Ausbildung klinischer Symptomatik [30]. Weder hinsichtlich der neurologischen Symptomatik 4 Wochen nach Behandlung noch hinsichtlich sekundärer Therapieziele bestanden signifikante Unterschiede zwischen den Gruppen [30]. Ein grenzwertig signifikant besseres Abschneiden der Kombinationstherapie wurde als nicht ausreichend zu deren Rechtfertigung empfunden und die Therapie mit IG $(0,4 \mathrm{~g} / \mathrm{kg}, 5 \mathrm{~d})$ aufgrund besserer Praktikabilität gegenüber Plasmapherese empfohlen.

- Chronisch inflammatorische demyelinisierende Polyradiculoneuropathie: Diese erworbene demyelinisierende Erkrankung ist durch progressive symmetrische proximale und distale Muskelschwäche bei A- oder Hyporeflexie und variabler sensorischer Mitbeteiligung gekennzeichnet. Die Ansprechrate der Plasmapherese ist bei dieser chronisch-rezidivierenden Erkrankung geringer als in den oben genannten Indikationen $[31,32]$.

\section{Purpura fulminans}

Diese Indikation ist nicht durch kontrollierte Studien gesichert. Mehrere Autoren berichten bei fulminanter Meningokokkensepsis, wo hohe Plasmakonzentrationen von Endotoxin und Cytokinen bestehen, über einer Verbesserung der Prognose nach Plasmapherese [33]. Jüngste Daten über die Infusion von Protein $\mathrm{C}$ dürften auch hier die Indikation zur Plasmapherese in Frage stellen [34]. Da im Plasma vorhandenes endotoxin binding peptide (EBP) die biologische Toxizität von Endotoxin erhöht, erscheint die Entfernung des Endotoxin-EBP Komplexes theoretisch sinnvoll. Eine Manipulation der Cytokinkaskade ohne suffiziente Einsicht in das komplexe pathophysiologische Geschehen läßt allerdings die Erfolgsaussichten einer unselektiven Behandlung wie der Plasmapherese gering erscheinen [33].

\section{Stoffwechselkrankheiten}

1. Thyreotoxische Krise: Durch Entfernung des Thyreoglobulins können Dauer und Schweregrad einer sonst therapierefraktären thyreotoxischen Krise vermindert werden [6].

2. Homozygote Hypercholesterinämie: Eine frühzeitige Plasmapherese kann zur Entfernung des fast ausschließlich intravaskulär vorliegenden LDL-Cholesterins bei Vorliegen klinischer Komplikationen (Angina pectoris, generalisierte Atherosklerose, Hyperviskosität) in Verbindung mit Gesamt-Cholesterin von $>350 \mathrm{mg} / \mathrm{dl}$ zur Verhinderung akuter ischämischer Komplikationen indiziert sein. Homozygote Patienten benötigen häufig eine spätere Fortführung spezifischer extrakorporaler Therapie (LDL-Apherese) [6].

3. HELLP-Syndrom: Die Indikationen zur Plasmapherese sind selten und werden auf schwere Entgleisungen des Fettstoffwechsels (TG >2000 mg/dl) beschränkt [6, 36].

\section{Intoxikationen}

Der klinische Einsatz der Plasmapherese beschränkt sich derzeit auf schwere Digitalisüberdosierung und Intoxikationen mit Paraquat, Parathion und die Frühphase der Amanitavergiftung. Bei schwerer Rhabdomyolyse kann durch frühzeitige Plasmapherese ein Tubulusschaden (CrushNiere) verhindert werden [6].

\section{Nicht gesicherte Indikationen}

Abstoßung nach Nierentransplantation: Die Indikation zur Plasmapherese wird kontrovers beurteilt. Lediglich für akute vaskuläre Abstoßungen scheint eine gewisse Wirksamkeit belegt [37]. Die meisten kontrollierten Studien sprechen jedoch gegen den Einsatz der Plasmapherese bei akuter und chronischer Abstoßungsreaktionen [3]. Die Entfernung des meist zuvor infundierten Antithymocytenglobulins erscheint auch ökonomisch fragwürdig. Anti-HLA-Antikörper bei Transplantationskandidaten: Die Entfernung von polyreaktiven Antikörpern vor Nierentransplantation ist eine prinzipiell vielversprechende Indikation $[38,39]$. Allerdings liegen noch keine eindeutigen kontrollierten Daten vor und zumindest eine Studie berichtet über eine erhöhte Inzidenz schwerer Infektionen [40]. Präoperative Plasmapherese in der Thoraxchirurgie: In dieser Indikation wird vor kardiochirurgischen Eingriffen thrombozytenreiches Plasma in einer Plasmapherese entnommen und postoperativ reinfundiert. Zwei kontrollierte Studien berichten über eine Reduktion des Transfusionsbedarfs, eine offensichtlich verbesserte Toleranz des kardiopulmonalen Bypass und eine Reduktion der Mediastinaldrain-Sekretion sowie des Transfusionsbedarfs an Erythrocyten und FFP [11, 14]. Herztrans- 
plantation bei positivem cross-match: In einer retrospektiven Analyse betrug die 2-Jahresüberlebensrate in der Plasmapheresegruppe $75 \%$, in der Immunsuppressionsgruppe 33\% [42]. Weitere nicht gesicherte Indikationen sind: Pruritus bei cholestatischen Lebererkrankungen, neonatale Hämolyse (IgM), systemischer Lupus erythematodes [16], Entfernung von anti- $\beta_{1}$-Rezeptor-Antikörpern zur hämodynamischen Verbesserung bei dilatativer Cardiomyopathie [43], fulminante akute disseminierte Encephalomyelitis [44], und akutes Leberversagen mit Encephalopathie [45] und Koagulopathie, wo ein reduzierter Transfusionsbedarf während Lebertransplantation erreicht wurde [46].

\section{Komplikationen der Plasmapherese}

Die Gesamt-Komplikationsrate der Plasmapherese liegt zwischen 1,6 und 25\% [2,3]. Schwere Komplikationen treten in $0,5-3 \%$, letale Zwischenfälle bei $0,05-0,1 \%$ auf $[5,47]$. Die am häufigsten genannten Komplikationen betreffen den Gefäßzugang und Nebenwirkungen des Substituats [2]. Jeder Entscheidung zur Plasmapherese sollte eine Nutzen-Risiko-Analyse vorausgehen. Abteilungen, welche mit den technischen Details der Plasmapherese nicht vertraut sind, sollten den Transfer von Patienten an ein Referenzzentrum erwägen.

\section{Komplikationen durch den Gefäßzugang}

Die im intensivmedizinischen Bereich bevorzugte Technik der Membranplasmapherese erfordert einen Blutfluß von ca. $100 \mathrm{ml} / \mathrm{min}$ und damit fast immer einen Dialysekatheter. Katheter der V. femoralis sollten aufgrund der hohen Thromboserate maximal 3 Tage belassen werden. Sowohl zu lange Katheter als auch Führungsdrähte können eine Perforation der V. cava superior und des rechten Atriums verursachen [48]. Dialysekatheter der V. jug. dextra sollten daher maximal $16 \mathrm{~cm}$ lang sein. Die Verwendung der V. subclavia ist mit einer höheren Infektionsrate und mit späteren Gefäßstenosen in Verbindung gebracht worden [49]. Punktionsassoziierte Komplikationen sind durch geeignete Technik und Verwendung von Sonographie reduzierbar [50]. Die Immunapherese kann unter Verwendung einer Zellzentrifugen-Separation auch über periphere Venen durchgeführt werden.

\section{Komplikationen durch Substituat}

Je höher die Plasmapheresefrequenz und das ausgetauschte Plasmavolumen, desto höher sind die Substituat-assoziierten Nebenwirkungen. Anaphylaktische Reaktionen und Hypokalziämie sind bei Plasmasubstitution besonders häu-
Tab. 2 Zusammensetzung von fresh frozen plasma [58]

\begin{tabular}{lc}
\hline Glucose & $535 \mathrm{mg} / \mathrm{dl}$ \\
Natrium & $172 \mathrm{mmol} / 1$ \\
Chlorid & $73 \mathrm{mmol} / 1$ \\
Kalium & $3.5 \mathrm{mmol} / 1$ \\
Calcium & $1,8 \mathrm{mmol} / 1$ \\
$\mathrm{HCO}_{3}$ & $15 \mathrm{mmol} / 1$ \\
GEW & $55 \mathrm{~g} / 1$ \\
Albumin & $30 \mathrm{~g} / 1$ \\
Cholesterin & $171 \mathrm{mg} / \mathrm{dl}$ \\
pH & 5,0 \\
\hline
\end{tabular}

fig; als langfristig gefährlichste Komplikation erscheinen Virusinfektionen [2, 5].

a) Albumin: Am häufigsten verwendetes Kolloid zur Substitution nach Plasmapherese. Gerinnungsstörung (PTZ-Abfall, PTT-Anstieg), Absinken der Fibrinogensynthese, Vasodilatation (bradykininartiger Faktor), allergische Reaktionen, pulmonale Extravasation v. a. bei Permeabilitätsschaden. Albumin kann problemlos teilweise oder ganz durch Plasmaexpander ersetzt werden; ob dies längerfristige klinische Folgen hat (Überladung des RES?) ist nicht bekannt [52].

b) Plasmaproteinlösungen: großer Spenderpool - Gefahr von Virusinfektionen da nur Kaltsterilisation möglich, allergische Reaktionen, Vasodilatation.

c) Fresh-frozen-plasma: Virusinfektionen, Citrat-Toxizität (Hypokalziämie, Arrhythmie, Alkalose) v.a. bei Lebererkrankungen mit gestörtem Citrat-Abbau; Alloimmunisierung, Anaphylaxie, hohe Osmolalität (Tabelle 2), Extravasation von Plasmaproteinen ins Interstitium, pulmonale Leukostase, Mikroaggregate, Komplementaktivierung und myokardiale Depression bei experimenteller Sepsis [53], ARDS [54]. An einigen Abteilungen erfolgt die Infusion von FFP bei Risikopatienten in die A. femoralis, wo eine Komplementaktivierung in der Mikrozirkulation weniger schwere Folgen hat als in der pulmonalen Zirkulation.

\section{Hämodynamische Komplikationen}

Die häufigste kardiovaskuläre Komplikation ist die Hypotonie. Aufgrund der onkotischen Wirkung des entfernten Plasma führen bereits geringe Minusbilanzen oder hypoonkotische Substituate zu Hypotonie, ferner lösen etliche Substituate eine periphere Vasodilatation aus (s.u.). Auch Arrhythmien (u.a. Asystolie) unter Citrat-Antikoagulation müssen hier erwähnt werden [51]. Bei Plusbilanzen kann auch eine akute Hypervolämie mit Lungenödem auftreten. 


\section{Komplikation durch Antikoagulation}

Die wichtigste Komplikation ist die Toxizität des NaCitrat, welches Hyperkalziämie, Arrhythmien und metabolische Alkalose auslösen kann [51]. Regelmäßiges Monitoring des ionisierten Calciums und prophylaktische Calciuminfusionen werden generell empfohlen, zumal Todesfälle durch Arrhythmien dokumentiert sind [51]. Die Notwendigkeit einer Antikoagulation und die zusätzliche gerinnungshemmende Nebenwirkung verschiedener Substituate kann gelegentlich die Blutungsneigung verstärken. Hauptsächliche Komplikationen der Antikoagulation unter Plasmapherese sind Blutungen an Einstichstellen, im Gastrointestinaltrakt, Lunge, ZNS, Retroperitoneum und Perikard. Diesbezüglich sind Patienten mit TTP besonders gefährdet.

\section{Komplikationen durch Entfernung plasmatischer Faktoren}

Gegenüber Immunsuppression ist die Rate an Infektionen in einigen Studien unter Plasmapherese vermehrt [40, 55]. Dies könnte neben Katheter-assoziierten Infektionen auf die Entfernung von Immunglobulinen und Komplement zurückgeführt werden [56, 57]. Jedenfalls sollte vor Beginn eines Plasmapheresezyklus jede Infektion sicher ausgeschlossen oder zumindest weitgehend antibiotisch therapiert werden. Vielfach wird auch eine Substitution von IgG nach Abschluß der Plasmapherese empfohlen, was aber nicht prospektiv abgesichert ist [2]. Fälle von klinisch relevanten Thrombosen nach Plasmapherese wurden v. a. durch Entfernung von AT-III aus dem Plasma erklärt [51], die Rate an signifikanten Blutungen infolge eines Faktorenmangels ist hingegen gering [2].

\section{Literatur}

1. Abel JJ, Rowntreee LG, Turner BB (1914) Plasma removal with return of corpuscles. J Pharmacol Exp Ther 5: 625-641

2. Reimann PM, Mason PD (1990) Plasmapheresis: Technique and complications. Intensive Care Med 16:3-10

3. Madore F, Lazarus JM, Brady HR (1996) Therapeutic plasma exchange in renal disease. J Am Soc Nephrol $7: 367-386$

4. Couriel D, Weinstein R (1994) Complications of therapeutic plasma exchange: A recent assessment. J Clin Apheresis 9:1-5

5. Franz HE, Hörl WH (Hrsg) (1997) Blutreinigungsverfahren. Technik und Klinik. 5. Auflage, Thieme, Stuttgart New York

6. Hakim RM, Siami GA (1994) Plasmapheresis. In: Handbook of Dialysis, $2^{\text {nd }}$ edition (Hrsg) Daugirdas JT, TS Ing. Boston, Little Brown and Company, pp 218-241

7. Schröder JO, Euler HH (1995) Plasmapherese bei Autoimmunerkrankungen. Die gelben Hefte 35: 137-145

8. Lockwood CM, et al. (1979) Reversal of impaired splenic function in patients with nephritis or vasculitis (or both) by plasma exchange. N Engl J Med 300: $524-530$

9. Rock GA, Shumak KH, Buskard NA, Blanchette VS, Kelton JG, Nair RC, Spasoff RA (1991) Comparison of plasma exchange with plasma infusion in the treatment of thrombotic thrombocytopenic purpura. Canadia Apheresis Study Group. N Engl J Med 325: $393-397$
10. Rivard GE, David M, Farrell C, Schwarz HP (1995) Treatment of purpura fulminans in meningococcemia with protein $\mathrm{C}$ concentrate. J Pediatr 126: 646-652

11. Menges T, Welters I, Wagner RM, Boldt J, Dapper F, Hempelmann G (1997) The influence of acute preoperative plasmapheresis on coagulation tests, fibrinolysis, blood loss and transfusion requirements in cardiac surgery. Eur J Cardiothorac Surg 11:557-563

12. Levy JB, Pusey CD (1997) Stil a role for plasma exchange in rapidly progressive glomerulonephritis? J of Nephrol 10:7-13

13. Pusey CD, Rees AJ, Evans DJ, Peters DK, Lockwood CM (1991) Plasma exchange in focal necrotizing glomerulonephritis without anti-GBM antibodies. Kidney Int 40:757-763

14. Lewis EJ, Hunsicker LG, Lan SP, Rohde RD, Lachin JM (1992) A controlled trial of plasmapheresis therapy in severe lupus nephritis. The Lupus Nephritis Collaborative Study Group. N Engl J Med 326: 1373-1379

15. Schröder JO, Euler HH (1995) Plasmapherese bei Autoimmunerkrankungen. Die gelben Hefte 35: 137-145

16. Euler HH, Schwab UM, Schroeder JO, Hasford J (1996) The Lupus Plasmapheresis Study Group: Rationale and updated interim report. Artif Organs 20:356-359

17. Appel GB, Valeri A (1994) The course and treatment of lupus nephritis. Annu Rev Med 45: 525-537

18. Misiani R, Tiraboschei G, Mingardi G, Mecca G (1987) Management of myeloma kidney: An anti-light-chain-approach. Am J Kidney Dis 10:28-33
19. Abdulkadyrov KM, Bessmeltsev SS (1996) Renal insufficiency in multiple myeloma: basic mechanisms in its development and methods for treatment. Ren Fail 18: 139-146

20. Sinico RA, Fornasieri A, Mosconi L, Florio G, Vecchi C (1985) Plasmapheresis in the treatment of essential mixed cryoglobulinemia nephropathy. Longterm follow up. Int Artif Organs 2: $19-22$

21. D'Amico Fornaseri A (1994) Cryoglobulinemic glomerulonephritis: A membranoproliferative glomerulonephritis induced by hepatitis $\mathrm{C}$ virus. $\mathrm{J}$ Am Kidney Dis 25:361-369

22. Bell WR, Hayden GB, Ness PM, Kickler TS (1991) Improved survival in thrombotic thrombocytopenic purpura - hemolytic uremic syndrome. New Engl J Med 325:398-403

23. Hoffkes HG, Heemann UW, Teschendorf C, Uppenkamp M, Philipp T (1995) Hyperviscosity syndrome: Efficacy and comparison of plasma exchange by plasma separation and cascade filtration in patients with immunocytoma of Waldenstrom's type. Clin Nephrol 43:335-338

24. Drachman DB (1996) Immunotherapy in neuromuscular disorders: Current and future strategies. Muscle \& Nerve 19: $1239-1251$

25. Gajdos P, Chevret S, Clair B, Tranchant C, Chastang C (1997) Clinical trial of plasma exchange and high-dose intravenous immunoglobulin in myasthenia gravis. Myasthenia Gravis Clinical Study Group. Ann Neurol 41:789-796 
26. National Institutes of Health: Consensus development conference statement: The utility of therapeutic plasmapheresis for neurological disorders. JAMA 256: $1333-1337$

27. McCann GM, Griffin JW, Cornblath DR, Mellits ED, Fisher RS, Quaskey SA (1988) Plasmapheresis and Guillain-Barré-syndrome: Analysis of prognostic factors and the effect of plasmapheresis. Ann Neurol 23: $347-353$

28. French Cooperative group in plasma exchange in Guillain Barré Syndrome. Efficacy of plasma exchange in Guillain Barré syndrome: role of replacement fluids. Ann Neurol 22: 753-761

29. Van der Meché FGA, Schmitz PIM, Dutch Guillain-Barré-Syndrom Study group (1992) A randomized trial comparing intravenous immune globuline and plasma exchange in GuillainBarré-Syndrom. New Engl J Med 326: $1123-1129$

30. The Plasma exchange/Sandoglobulin Guillain-Barré Syndrome Trial group. Randomised trial of plasma exchange, intravenous immunoglobulin, and combined treatments in GuillainBarré-Syndrome. Lancet 349: $225-230$

31. Van der Meché FGA, Vermeulen M, Busch HFM (1989) Chronic inflammatory demyelinating polyneuropathy: conduction failure before and during immunoglobulin or plasma therapy. Brain 112: 1563-1571

32. Mendell JR (1993) Chronic inflammatory demyelinating polyradiculopathy. Annu Rev Med 44:211-219

33. Scharfman WB, Tillotson JR, Taft EG, Wright E (1979) Plasmapheresis for meningocoocemia with disseminated intravascular coagulation. N Engl J Med 300: $1277-1279$

34. Smith OP, White B, Vaughan D, Rafferty M, Claffey L, Lyons B, Casey W (1998) Use of protein-C concentrate, heparin, and haemodiafiltration in meningococcus-induced purpura fulminans. Lancet 350: 1590-1593

35. Reinke P (1996) Plasmapheresis in the therapy of septic disease. Int J Artif Organs 19: 127-128

36. Bambauer R, Schiel R, Latza R, Klinkmann J (1996) Treatment of severe hyperlipidemia: Six years' experience with low-density lipoprotein apheresis. Artif Organs 20:336-339
37. Bonomini V, Vangelista A, Frasca GM, Di Felice A, Liviano-D'Arcangelo G (1985) Effects of plasmapheresis in renal transplantation. A controlled study. Trans Am Soc Artif Intern Organs 31:698-703

38. Taube D, Palmer A, Welsh K, Bewick M, Snowden S, Thick M (1989) Removal of anti-HLA antibodies prior to transplantation: An effective and successful strategy for highly sensitized renal allograft recipients. Transplant Proc 21:694-695

39. Alarabi A, Backman U, Wikstrom B, Sjoberg O, Tufveson G (1997) Plasmapheresis in HLA-immunosensitized patients prior to kidney transplantation. Int J Artif Organs 20:51-56

40. Frasca GM, Martella D, Vangelista A, Bonomini V (1991) Ten years experience with plasma exchange in renal transplantation. Int J Artif Organs 14:51-55

41. Armellin FG, Sorbara C, Bonato R Pittarello D, Dal-Cero P, Giron G (1997) Intraoperative plasmapheresis in cardiac surgery. J Cardiothorac Vasc Anesth 11:13-17

42. Ratkovec RM, Hammond EH, O'Connell JB, Bristow MR, DeWitt CW, Richenbacher WE, Millar RC, Renlund DG (1992) Outcome of cardiac transplant recipients with a positive donor-specific crossmatch - Preliminary results with plasmapheresis. Transplantation 54:651-655

43. Dorffel WV, Felix SB, Wallukat G, Brehme S, Bestvater K, Hofmann T, Kleber FX, Baumann G, Reinke P (1997) Short-term hemodynamic effects of immunoadsorption in dilated cardiomyopathy. Circulation 95: 19791980

44. Kanter DS, Horensky D, Sperling RA, Kaplan JD, Malachowski ME, Churchill WH Jr (1995) Plasmapheresis in fulminant acute disseminated encephalomyelitis. Neurology 45: 824-827

45. Buckner CD, Clift RA, Wolwiler W, et al. (1977) Plasma exchange in patients with fulminant hepatic failure. Arch Int Med 132: 487-492

46. Hackl W, Zadrobilek E, Mauritz W, Längle F, Höcker P, Sporn P (1989) Präoperativer Plasmaaustausch zur Therapie plasmatischer Gerinnungsstörungen vor Lebertransplantation. Anästhesist 38:539-543

47. Karmochkine M, Bussel A, Leon A, Jarrousse B, Baudelot J, Guillevin L (1994) Long-term plasma exchange. Analysis of indications, outcome and side effects. Ann Med Interne Paris 145: $373-548$
48. Konner K (1997) Probleme des geeigneten Gefäßzuganges bei der Anwendung von Nierenersatzverfahren bei akutem Nierenversagen. Intensivmed 34: 569-576

49. Bambauer R, Inniger R, Pirrung KJ, Schiel R, Dahlem R (1994) Complications and side effects associated with large-bore catheters in the subclavian and internal jugular veins. Artif Organs 18:318-321

50. Skolnick ML (1994) The role of sonography in the placement and management of jugular and subclavian central venous catheters. AJR-Am J Roentgenol 163:291-295

51. The Lancet (1982) Hazards of apheresis (editorial). Lancet 1982: $1025-1026$

52. Le Conte P, Nicolas F, Adjou C, N'Guyen JM, Billaud E, Moreau P (1997) Replacement fluids in plasmapheresis: Cross-over comparative study. Intensive Care Med 23: $342-344$

53. Busund R, Balteskard L, Ronning G, Hogasen K, Revhaug A (1995) Fatal myocardial depression and circulatory collapse associated with complement activation induced by plasma infusion in severe porcine sepsis. Acta Anesthesiol Scand 39: 100-108

54. Boogaerts MA, Roelant C, Goosens W, Verwilghen RL (1986) Complement activation and adult respiratory distress syndrome during intermittent flow apheresis procedure. Transfusion 26: $82-87$

55. Wing EJ, Bruns FJ, Fraley DS, Segel DP, Sheldon A (1980) Infectious complications with plasmapheresis in rapidly progressive glomerulonephritis. JAMA 244: 243-247

56. Keller AJ, Urbaniak SJ (1978) Intensive plasma exchange on the cell separator: Effects on serum immunoglobulins and complement components. Br J Haematol 38: 531 - 540

57. Sultan Y, Bussel A, Maisonneuve P, Poupeney M, Sitty X, Gajdos P (1979) Potential danger of thrombosis after plasma exchange in the treatment of patients with immune disease. Transfusion 19:588-593

58. Evalenko P, Deloof T, Peeters J (1986) Composition of fresh frozen plasma. Crit Care Med 14: 145-146 